\title{
LIMIT DISTRIBUTIONS FOR QUEUES AND RANDOM ROOTED TREES ${ }^{1}$
}

\author{
LAJOS TAKÁCS \\ Case Western Reserve University \\ Cleveland, Ohio 44106 USA
}

\begin{abstract}
In this paper several limit theorems are proved for the fluctuations of the queue size during the initial busy period of a queuing process with one server. These theorems are used to find the solutions of various problems connected with the heights and widths of random rooted trees.
\end{abstract}

Key words: Single-server queues, queue size, random trees, heights and widths.

AMS (MOS) subject classifications: $\quad 60 \mathrm{~K} 25,05 \mathrm{C} 05$.

\section{INTRODUCTION}

There is an intrinsic relationship between queuing processes and rooted trees. Most of the results of this paper are built on this relationship. We shall study the stochastic behavior of the fluctuations of the queue size during the initial busy period of a single-server queuing process and make use of the results obtained for the solutions of various problems connected with the heights and widths of random rooted trees. We consider a queuing process with one server. It is supposed that initially, when the server starts working, the first customer is already waiting for service. The server serves this customer and all the new customers in order of arrival as long as they keep coming. Denote by $i_{1}, i_{2}, \ldots$ the number of arrivals during the first, second, ... service times respectively. If there are no more customers to serve, the initial busy period ends. The initial busy period consists of $n$ services if and only if

$$
i_{1}+i_{2}+\cdots+i_{n}=n-1
$$

and

\footnotetext{
${ }^{1}$ Received: May, 1993. Revised: July, 1993.
} 


$$
i_{1}+i_{2}+\cdots+i_{r} \geq r \text { for } 1 \leq r \leq n-1 .
$$

If the initial busy period consists of $n$ services, let us associate the following graph with the queuing process considered: The graph has vertex set $(1,2, \ldots, n)$ and vertices $r$ and $s$ where $1 \leq r<s \leq n$ are joined by an edge if and only if the $s$ th customer arrives during the service time of the $r$ th customer. Evidently, the graph is a rooted tree with vertex set $(1,2, \ldots, n)$, vertex 1 being the root of the tree. Different queuing processes yield different trees.

If in the queuing process, the number of arrivals during the successive service times are random variables, which we shall denote by $\nu_{1}, \nu_{2}, \ldots$, and if the initial busy period consists of $n$ services, then the corresponding graph is a random rooted tree with $n$ vertices. We shall assume that $\left\{\nu_{r}\right\}$ is a sequence of independent and identically distributed discrete random variables with distribution

$$
P\left\{\nu_{r}=j\right\}=p_{j}
$$

for $j=0,1,2, \ldots$. We obtain various models of random rooted trees by choosing the distribution $\left\{p_{j}\right\}$ in a suitable way.

In what follows we shall use some combinatorial theorems which are the generalizations of the classical ballot theorem of Bertrand [4]. We shall express the various limit distributions as the distributions of some functionals defined on the Brownian excursion $\left\{\eta^{+}(t), 0 \leq t \leq 1\right\}$. After studying the stochastic behavior of the initial busy period for various queuing processes, we derive some limit theorems for the heights and widths of random rooted trees.

The results of this paper are the extensions of Takács [40], [42], [43], and [44] and were presented at the International Conference on Random Mappings, Partitions, and Permutations, Los Angeles, January 1992. See Takács [45].

\section{COMBINATORIAL THEOREMS}

Let $\nu_{1}, \nu_{2}, \ldots, \nu_{r}, \ldots$ be independent discrete random variables which take on nonnegative integers only. Write $N_{r}=\nu_{1}+\nu_{2}+\cdots+\nu_{r}$ for $r \geq 1$ and $N_{0}=0$.

Theorem 1: We have

$$
P\left\{N_{r}<r \text { for } 1 \leq r \leq n \text { and } N_{n}=n-k\right\}=\frac{k}{n} P\left\{N_{n}=n-k\right\}
$$


for $0 \leq k \leq n$ and $n=1,2, \ldots$

Proof: This theorem is a generalization of the classical ballot theorem of Bertrand [4]. For its proof, see Takács [35], [36], and [39].

Define

$$
\rho(k)=\inf \left\{r: r-N_{r}=k, r \geq 0\right\}
$$

for $k=0,1,2, \ldots$. If $r-N_{r}<k$ for all $r \geq 0$, then $\rho(k)=\infty$.

Theorem 2: We have

$$
P\{\rho(k)=n\}=\frac{k}{n} P\left\{n-N_{n}=k\right\}
$$

for $n \geq 1$ and $k \geq 0$.

Proof: If $k>n$, then both sides of (6) are 0 . If $0 \leq k \leq n$, and $n \geq 1$, then by Theorem 1 ,

$$
\begin{aligned}
P\{\rho(k)=n\}= & P\left\{r-N_{r}<k \text { for } 0 \leq r<n \text { and } N_{n}=n-k\right\}= \\
& P\left\{N_{n}-N_{r}<n-r \text { for } 0 \leq r<n \text { and } N_{n}=n-k\right\}= \\
& P\left\{N_{i}<i \text { for } 1 \leq i \leq n \text { and } N_{n}=n-k\right\}=\frac{k}{n} P\left\{N_{n}=n-k\right\} .
\end{aligned}
$$

Theorem 3: Let $f\left(k_{1}, k_{2}, \ldots, k_{n}\right)$ be a symmetric function of the variables $k_{1}, k_{2}, \ldots, k_{n}$ where $k_{i}=0,1,2, \ldots$ Then

$$
\begin{gathered}
\sum_{\begin{array}{c}
k_{1}+k_{2}+\cdots+k_{n}=k \\
k_{1}+\cdots+k_{r}<r \text { for } 1 \leq r \leq n
\end{array}} f\left(k_{1}, k_{2}, \ldots, k_{n}\right) \\
=\frac{n-k}{n} \sum_{k_{1}+k_{2}+\cdots+k_{n}=k} f\left(k_{1}, k_{2}, \ldots, k_{n}\right)
\end{gathered}
$$

for $0 \leq k \leq n$.

Proof: We can prove (8) by mathematical induction on $n$ if we take into consideration that in (8) $k_{n}$ may take on the values $0,1, \ldots, k$ where $k \leq n$. As an alternative we can prove (8) by the repeated applications of Theorem 1. We note that (8) still remains valid if we assume only that the function 
$f\left(k_{1}, k_{2}, \ldots, k_{n}\right)$ is invariant under the $n$ cyclic permutations of $\left(k_{i}, k_{2}, \ldots, k_{n}\right)$.

If, in particular,

$$
f\left(k_{1}, k_{2}, \ldots, k_{n}\right)=g\left(k_{1}\right) g\left(k_{2}\right) \ldots g\left(k_{n}\right),
$$

then Theorem 3 is applicable and in (8)

$$
\sum_{k_{1}+k_{2}+\cdots+k_{n}=k} f\left(k_{1}, k_{2}, \ldots, k_{n}\right)=\text { Coeff. of } x^{k} \text { in }\left(\sum_{i=0}^{\infty} g(i) x^{i}\right)^{n} .
$$

\section{THE BROWNIAN EXCURSION}

The Brownian excursion process $\left\{\eta^{+}(t), 0 \leq t \leq 1\right\}$ is a Markov process for which $P\left\{\eta^{+}(0)=0\right\}=P\left\{\eta^{+}(1)=0\right\}=1$ and $P\left\{\eta^{+}(t) \geq 0\right\}=1$ for $0 \leq t \leq 1$. If $0 \leq t \leq 1$, then $\eta^{+}(t)$ has a density function $f(t, x)$. Obviously, $f(t, x)=0$ for $x \leq 0$. If $0<t<1$ and $x>0$, then

$$
f(t, x)=\frac{2 x^{2}}{\sqrt{2 \pi t^{3}(1-t)^{3}}} e^{-x^{2} /(2 t(1-t))} .
$$

If $0<t<u<1$, then the random variables $\eta^{+}(t)$ and $\eta^{+}(u)$ have a joint density function $f(t, x ; u, y)$. We have $f(t, x ; u, y)=0$ if $x \leq 0$ or $y \leq 0$. If $0<t<u<1$ and $x>0, y>0$, then

$f(t, x ; u, y)$

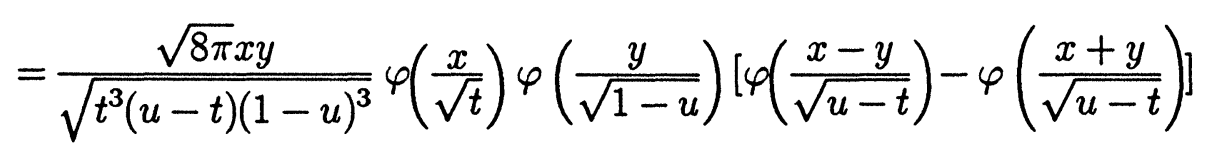

where

$$
\varphi(x)=\frac{1}{\sqrt{2 \pi}} e^{-x^{2} / 2}
$$

is the normal density function. For the properties of the Brownian excursion process we refer to Lévy [24] and [25], Itô and McKean, [13], Chung [7] and Takács [40].

For the Brownian excursion $\left\{\eta^{+}(t), 0 \leq t \leq 1\right\}$ we define

$$
\omega^{+}=\int_{0}^{1} \eta^{+}(t) d t
$$


and $\tau^{+}(\alpha)$ for $\alpha \geq 0$ as the local time at level $\alpha$, that is,

$$
\tau^{+}(\alpha)=\lim _{\epsilon \rightarrow 0} \frac{1}{\epsilon} \text { measure }\left\{t: \alpha \leq \eta^{+}(t)<\alpha+\epsilon, 0 \leq t \leq 1\right\} .
$$

Let

$$
\begin{gathered}
P\left\{\sup _{0 \leq t \leq 1} \eta^{+}(t) \leq x\right\}=F(x), \\
P\left\{\omega^{+} \leq x\right\}=W(x),
\end{gathered}
$$

and

$$
P\left\{\tau^{+}(\alpha) \leq x\right\}=G_{\alpha}(x) .
$$

We note that $G_{\alpha}(0)=F(\alpha)$ for $\alpha>0$.

If $x>0$, then

$$
F(x)=\sum_{j=-\infty}^{\infty}\left(1-4 j^{2} x^{2}\right) e^{-2 j^{2} x^{2}}=\frac{\sqrt{2} \pi^{5 / 2}}{x^{3}} \sum_{j=0}^{\infty} j^{2} e^{-j^{2} \pi^{2} /\left(2 x^{2}\right)}
$$

and $F(x)=0$ for $x \leq 0$. In 1952, Gnedenko and Studnev [11] determined $F^{\prime}(x)$ in the context of order statistics. See also Takács [34] and Kennedy [20]. The moments

$$
\mu_{r}=\int_{0}^{\infty} x^{r} d F(x)
$$

exist for $r \geq 0$ and $\mu_{0}=1, \mu_{1}=\sqrt{\pi / 2}, \mu_{2}=\pi^{2} / 6, \mu_{3}=3 \sqrt{\pi} \zeta(3) / \sqrt{8}, \mu_{4}=\pi^{4} / 30$. For $r>1$,

$$
\mu_{r}=2(r-1) \Gamma\left(\frac{r}{2}+1\right) \zeta(r) / 2^{r / 2}
$$

where

$$
\zeta(r)=\sum_{n=1}^{\infty} \frac{1}{n^{r}}
$$

is the Riemann zeta function.

If $x>0$, then

$$
W(x)=\frac{\sqrt{6}}{x} \sum_{k=1}^{\infty} e^{-v_{k}} v_{k}^{2 / 3} U\left(1 / 6,4 / 3, v_{k}\right)
$$

where $U(a, b, x)$ is the confluent hypergeometric function,

$$
v_{k}=2 a_{k}^{3} /\left(27 x^{2}\right),
$$

and $z=-a_{k}(k=1,2, \ldots)$ are the zeros of the Airy function $A i(z)$ arranged so 
that $0<a_{1}<a_{2}<\ldots<a_{k}<\ldots$. If $x \leq 0$, then $W(x)=0$. The moments

$$
M_{r}=\int_{0}^{\infty} x^{r} d W(x)
$$

exist for $r \geq 0$ and $M_{0}=1, M_{1}=\sqrt{\frac{\pi}{8}}, M_{2}=\frac{5}{12}, M_{3}=\frac{15}{32} \sqrt{\frac{\pi}{8}}, M_{4}=\frac{221}{1008}$. See L. Takács [40].

If $x \geq 0$, then

$$
G_{\alpha}(x)=1-2 \sum_{j=1}^{\infty} \sum_{k=0}^{j-1}\left(\begin{array}{c}
j-1 \\
k
\end{array}\right) e^{-(x+2 \alpha j)^{2} / 2}(-x)^{k} H_{k+2}(x+2 \alpha j) / k !
$$

where $H_{0}(x), H_{1}(x), \ldots$ are the Hermite polynomials defined by

$$
H_{n}(x)=n ! \sum_{j=0}^{[n / 2]} \frac{(-1)^{j} x^{n-2 j}}{2^{j} j !(n-2 j) !}
$$

If $x<0$, then $G_{\alpha}(x)=0$. The moments

$$
\mu_{r}(\alpha)=\int_{0}^{\infty} x^{r} d G_{\alpha}(x)
$$

exist for $r \geq 0$. We have $\mu_{0}(\alpha)=1, \mu_{1}(\alpha)=4 \alpha e^{-2 \alpha^{2}}$ and

$$
\mu_{2}(\alpha)=4\left(e^{-2 \alpha^{2}}-e^{-8 \alpha^{2}}\right) \text {. }
$$

See Takács [41] and [42].

From the results of this paper we can draw the conclusion that

$$
\begin{aligned}
& P\left\{\sup _{\alpha \geq 0} \tau^{+}(\alpha) \leq 2 x\right\}=F(x), \\
& P\left\{\frac{1}{2} \int_{0}^{\infty}\left[\tau^{+}(\alpha)\right]^{2} d \alpha \leq x\right\}=W(x)
\end{aligned}
$$

and

$$
P\left\{\int_{0}^{\infty} \alpha \tau^{+}(\alpha) d \alpha \leq x\right\}=W(x)
$$

Accordingly, the random variables $\sup _{0 \leq t \leq 1} \eta^{+}(t)$ and $\frac{1}{2} s u p_{\alpha \geq 0^{+}} \tau^{+}(\alpha)$ have exactly the same distribution function $F(x)$. For a direct proof of this result see Jeulin [14], p. 264.

Moreover, the random variables 


$$
\int_{0}^{1} \eta^{+}(t) d t, \quad \frac{1}{2} \int_{0}^{\infty}\left[\tau^{+}(\alpha)\right]^{2} d \alpha \text { and } \int_{0}^{\infty} \alpha \tau^{+}(\alpha) d \alpha
$$

also have the same distribution function $W(x)$.

\section{SINGLE-SERVER QUEUES}

Let us suppose that in the time interval $(0, \infty)$ customers arrive at random at a counter and are served singly by one server in order of arrival. It is assumed tat the server starts working at time $t=0$ and at that time $i$ $(i=1,2, \ldots)$ customers are already waiting for service. The initial $i$ customers are numbered $1,2, \ldots, i$ and the customers arriving subsequently are numbered $i+1, i+2, \ldots$ in the order of their arrivals. Denote by $\nu_{r}$ the number of customers arriving during the service time of the $r$ th customer. This queuing model will be characterized by the initial queue size $i$ and the sequence of random variables $\nu_{1}, \nu_{2}, \ldots, \nu_{r}, \ldots$. Throughout this paper we use the abbreviation $N_{r}=\nu_{1}+\nu_{2}+\cdots+\nu_{r}$ for $r=1,2, \ldots$ and $N_{0}=0$.

Denote by $\zeta_{r}(r=1,2, \ldots)$ the number of customers in the system immediately after the $r$ th service ends and write $\zeta_{0}=i$. We have

$$
\zeta_{r}=\left[\zeta_{r-1}-1\right]^{+}+\nu_{r}
$$

for $r \geq 1$ where $[x]^{+}=x$ if $x \geq 0$ and $[x]^{+}=0$ if $x<0$.

Following Kendall [18], we say that the initial $i$ customers in the queue form the 0th generation. The customers (if any) arriving during the total service time of the initial $i$ customers form the first generation. Generally, the customers (if any) arriving during the total service time of the customers in the $(r-1)$ th generation form the $r$ th generation for $r=1,2, \ldots$. Denote by $\xi(r)$ $(r=1,2, \ldots)$ the number of customers in the $r$ th generation. If $\xi(r)=0$ for some $r \geq 1$, then $\xi(r+1)=\xi(r+2)=\ldots=0$. Define

$$
\theta(i)=\max \{r: \xi(r)>0\}
$$

If $\xi(r)>0$ for all $r \geq 0$, then $\theta(i)=\infty$.

The time of the server consists of alternating busy periods and idle periods. Denote by $\rho(i)$ the number of customers served in the initial busy 
period in the case where the initial queue size is $i(i=1,2, \ldots)$. Obviously,

if $1 \leq i \leq n$.

$$
P\{\rho(i)=n\}=P\left\{N_{r}>r-i \text { for } i \leq r<n \text { and } N_{n}=n-i\right\}
$$

In what follows we assume that $\nu_{1}, \nu_{2}, \ldots, \nu_{r}, \ldots$ is a sequence of independent and identically distributed random variables for which

$$
P\left\{\nu_{r}=j\right\}=p_{j}
$$

if $j=0,1,2, \ldots$ where $p_{j} \geq 0$, and

Define

$$
\sum_{j=0}^{\infty} p_{j}=1
$$

$$
d=\operatorname{gcd}\left\{j: p_{j}>0\right\}
$$

and

If $a<\infty$, define $\sigma \geq 0$ by

$$
a=\sum_{j=0}^{\infty} j p_{j}
$$

$$
\sigma^{2}=\sum_{j=0}^{\infty}(j-a)^{2} p_{j}
$$

If $\nu_{1}, \nu_{2}, \ldots, \nu_{r}, \ldots$ are independent and identically distributed random variables, then in (36) we can replace $\nu_{1}, \nu_{2}, \ldots, \nu_{n}$ by $\nu_{n}, \nu_{n-1}, \ldots, \nu_{1}$ respectively, without changing the probability. Thus we obtain

$$
P\{\rho(i)=n\}=P\left\{N_{r}<r \text { for } 1 \leq r \leq n \text { and } N_{n}=n-i\right\}
$$

for $1 \leq i \leq n$. By Theorem 2 we have

$$
P\{\rho(i)=n\}=\frac{i}{n} P\left\{N_{n}=n-i\right\}
$$

for $1 \leq i \leq n$. Each possible value of $\rho(i)$ has the form $n=s d+i$ where $s=0,1,2, \ldots$, and actually, $P\{\rho(i)=n\}>0$ if $n=s d+i$ and $s$ is sufficiently large.

In what follows, we are interested in finding the probabilities

$$
F_{m}(n \mid i)=P\left\{\zeta_{r} \leq m \text { for } 0 \leq r \leq n \text { and } \rho(i)=n\right\}
$$

and

$$
G_{m}(n \mid i)=P\{\xi(r) \leq m \text { for } 0 \leq r \leq \theta(i) \text { and } \rho(i)=n\}
$$


for $1 \leq i \leq n$ and $1 \leq m \leq n$ and their asymptotic behavior as $n \rightarrow \infty$ and $m \rightarrow \infty$.

For each $m \geq 1$ we can determine (44) and (45) recursively for $n=1,2, \ldots$, and $1 \leq i \leq n$.

If we take into consideration that in the queuing process the number of arrivals during the first service may be $k=0,1,2, \ldots$, then we obtain that

$$
F_{m}(n \mid i)=\sum_{k=0}^{\min (m, n-i)} p_{k} F_{m}(n-1 \mid k+i-1)
$$

for $1 \leq i<n$ and $1 \leq i \leq m$ where $F_{m}(n \mid i)=0$ if $i>m$ or $i>n$, and

$$
F_{m}(n \mid n)=P\left\{N_{n}=0\right\}
$$

for $1 \leq n \leq m$.

We note that if $p_{j}=q p^{j}(j=0,1,2, \ldots)$ where $p>0, q>0$ and $p+q=1$, then $F_{m}(n \mid i)=H_{m}(n \mid i)$ where

$$
H_{m}(n \mid i)=p^{n-i} q^{n} \sum_{j}\left\{\left(\begin{array}{c}
2 n-i-1 \\
n-1+j(m+2)
\end{array}\right)-\left(\begin{array}{c}
2 n-i-1 \\
n+j(m+2)
\end{array}\right)\right\}
$$

for $1 \leq i<n$ and $1 \leq i \leq m$.

If $p_{0}=q, p_{2}=p$ where $p>0, q>0, p+q=1$ and $p_{j}=0$ otherwise, then necessarily $n=i+2 s(s=0,1,2, \ldots)$ and $F_{m}(n \mid i)=H_{m}^{*}(n \mid i)$ where

$$
H_{m}^{*}(n \mid i)=p^{s} q^{n-s} \sum_{j}\left\{\left(\begin{array}{c}
n-1 \\
s+j(m+2)
\end{array}\right)-\left(\begin{array}{c}
n-1 \\
s-1+j(m+2)
\end{array}\right)\right\}
$$

for $1 \leq i<n$ and $1 \leq i \leq m$. Both (48) and (49) can be proved simply by using the reflection principle for random walks. See Takács [34] and [37]. We note that

$$
H_{m}^{*}(2 n-i \mid i)=H_{m}(n \mid i)
$$

where the left-hand side is defined by (49) and the right-hand side by (48).

In the same way as (46) we obtain that

$$
G_{m}(n \mid i)=\sum_{k=1}^{\min (m, n-i)} P\left\{N_{i}=k\right\} G_{m}(n-i \mid k)
$$

for $1 \leq i<n$ and $1 \leq i \leq m$ where $G_{m}(n-i \mid i)=0$ if $i>m$ or $i>n$, and 


$$
G_{m}(n \mid n)=P\left\{N_{n}=0\right\}
$$

if $1 \leq n \leq m$. Starting from the initial conditions, we can determine $G_{m}(n \mid m)$ for $n=1,2, \ldots$ and $1 \leq i \leq n$. Also for fixed $m$ and $n$ we can consider (51) as a system of $m$ linear equations for the determination of $G_{m}(n \mid i)$ for $i=1,2, \ldots, m$. If the $\left(m_{2}+1\right)$ roots $\lambda=\lambda_{j}(m)\left(j=1,2, \ldots,\left({ }^{m}+1\right)\right)$ of the equation

$$
\operatorname{Det}\left[\lambda^{i} \delta_{i, k}-P\left\{N_{i}=k\right\}\right]_{i, k=1,2, \ldots, m}=0
$$

are distinct, then

$$
G_{m}(n \mid i)=\sum_{j=0}^{(m+1)} a_{i, j}(m)\left[\lambda_{j}(m)\right]^{n}
$$

for $n=1,2, \ldots$ where $a_{i, j}(m)$ does not depend on $n$. In (53) $\delta_{i, k}=0$ if $i \neq k$ and $\delta_{i, k}=1$ if $i=k$.

\section{THE PROCESS $\left\{\zeta_{r}, r \geq 0\right\}$}

We can express (44) in the following form:

$$
F_{m}(n \mid i)=P\left\{0<i+N_{r}-r \leq m \text { for } 0 \leq r \leq n \text { and } i+N_{n}-n=0\right\}
$$

for $1 \leq i<n$ and $1 \leq i \leq m$. In (55) $N_{r}-r=\left(\nu_{1}-1\right)+\left(\nu_{2}-1\right)+\ldots+\left(\nu_{r}-1\right)$ is the $r$ th partial sum of independent and identically distributed random variables and $\rho(i)=n$ if and only if $r=n$ is the smallest $r=1,2, \ldots$ for which $i+N_{r}-r=0$. Accordingly,

$$
\begin{array}{r}
\boldsymbol{P}\left\{\zeta_{r} \leq m \text { for } 0 \leq r \leq n \mid \rho(i)=n\right\} \\
=\boldsymbol{P}\left\{i+N_{r}-r \leq m \text { for } 1 \leq r \leq n \mid \rho(i)=n\right\}
\end{array}
$$

provided that $P\{\rho(i)=n\}>0$. If $n=s d+i \quad(s=0,1,2, \ldots)$ and $s$ is sufficiently large, then $P\{\rho(i)=n\}>0$. If we assume that in (40) $a=1$ and in (41) $0<\sigma<\infty$, then

$$
\left\{\frac{i+N_{[n t]}-[n t]}{\sigma \sqrt{n}}, 0 \leq t \leq 1 \mid \rho(i)=n\right\} \Rightarrow\left\{\eta^{+}(t), 0 \leq t \leq 1\right\},
$$

where $\left\{\eta^{+}(t), 0 \leq t \leq 1\right\}$ is the Brownian excursion process. The meaning of (57) is that if $n \rightarrow \infty$ the stochastic process on the left-hand side, given the condition 
$\rho(i)=n$, converges weakly to the Brownian excursion process. This is a consequence of a theorem of Kaigh [15] and [16]. Previously, Belkin [2] and [3] considered a variant of (57) in which the condition is $\rho(i)>n$. See also Iglehart [12] and Bolthausen [5].

Theorem 4. If $a=1,0<\sigma<\infty$ and $n=s d+1(s=0,1,2, \ldots)$, then

$$
\lim _{n \rightarrow \infty} P\left\{\max \left(\zeta_{0}, \zeta_{1}, \ldots, \zeta_{n}\right) \leq x \sigma \sqrt{n} \mid \rho(i)=n\right\}=F(x)
$$

where $F(x)$ is defined by (16).

Proof: Since the supremum is a continuous functional on the Brownian excursion process, (58) immediately follows from (57).

The limit theorem (57) suggests that if

for $r \geq 2$, then

$$
\sum_{j=0}^{\infty} j^{r} p_{j}<\infty
$$

$$
\lim _{n \rightarrow \infty} E\left\{\left(\frac{\max \left(\zeta_{0}, \zeta_{1}, \ldots, \zeta_{n}\right)}{\sigma \sqrt{n}}\right)^{r} \mid \rho(i)=n\right\}=\mu_{r}
$$

where $\mu_{r}$ is defined by (20). This statement is indeed true in the two particular cases covered by (48) and (49). The proof for $r=1$ follows from the results of de Bruijn, Knuth and Rice [8], and for $r>1$, from the results of Kemp [17].

Theorem 5: If $a=1,0<\sigma<\infty$ and $n=s d+1(s=0,1,2, \ldots)$, then

$$
\lim _{n \rightarrow \infty} P\left\{\zeta_{1}+\zeta_{2}+\ldots+\zeta_{n} \leq x \sigma n^{3 / 2} \mid \rho(i)=n\right\}=W(x)
$$

where $W(x)$ is defined by (17).

Proof: Since the integral is a continuous functional on the Brownian excursion process, (61) immediately follows from (57).

\section{THE PROCESS $\{\xi(r), r \geq 0\}$}

Under the assumption that $\nu_{1}, \nu_{2}, \ldots, \nu_{r}, \ldots$ are independent and identically distributed random variables, the sequence $\{\xi(r), r \geq 0\}$ is a branching process. We can imagine that in a population initially we have $i(i=1,2, \ldots)$ progenitors and in each generation each individual reproduces, independently of the others, and has probability $p_{j}(j=0,1,2, \ldots)$ of giving rise to $j$ descendants in the 
following generation. Then $\xi(r)$ can be interpreted as the number of individuals in the $r$ th generation $(r=0,1,2, \ldots)$. Obviously,

$$
\rho(i)=\sum_{r \geq 0} \xi(r)
$$

that is, $\rho(i)$ is the total number of individuals (total progeny) in the branching process if $\xi(0)=i$. Possibly, $\rho(i)=\infty$.

Evidently, $\{\xi(r), 0 \leq r \leq \theta(i)\}$ is a subsequence of $\left\{\zeta_{r}, 0 \leq r \leq \rho(i)\right\}$, and $\xi(r)=\zeta_{s}$ if $s=\xi(0)+\xi(1)+\ldots+\xi(r-1)$ for $r \geq 1$. This implies that if $\rho(i)=n$, then

$$
0 \leq \max _{0 \leq r \leq n} \zeta_{r}-\max _{0 \leq r \leq \theta(i)} \xi(r) \leq \quad \max _{|j-k| \leq \max \xi(r)(r \geq 0)}\left|\zeta_{j}-\zeta_{k}\right|
$$

and

$$
\left|\sum_{r=0}^{n} \zeta_{r}-\sum_{r=0}^{\theta(i)}[\xi(r)]^{2}\right| \leq n \quad \underset{|j-k| \leq \max \xi(r)(r \geq 0)}{\max }\left|\zeta_{j}-\zeta_{k}\right| .
$$

If $r \geq 1$, if $a=1$, if $0<\sigma<\infty$, if (59) holds, and if $n=s d+1$ $(s=0,1,2, \ldots)$, then for any $\epsilon>0$ we have

$$
\left|E\left\{\left(\begin{array}{c}
\xi(m) \\
r
\end{array}\right) \mid \rho(i)=n\right\}-\frac{1}{r !}\left(\frac{\sigma \sqrt{n}}{2}\right)^{r} \mu_{r}\left(\frac{m \sigma}{2 \sqrt{n}}\right)\right|<\epsilon n^{3 / 2} m^{r-3}
$$

for sufficiently large $m$ and $n$ where $\mu_{r}(\alpha)$ is defined by (28). We have $\mu_{0}(\alpha)=1$, $\mu_{1}(\alpha)=4 \alpha e^{-2 \alpha^{2}}$ and

for $r \geq 2$ and $\alpha>0$, and

$$
\mu_{r}(\alpha)=2^{r+1} r ! \alpha^{r} \int_{0}^{r-1}(1+x)^{-2 \alpha^{2}(1+x)^{2}} g_{r-1}(x) d x
$$

$$
g_{r-1}(x)=\sum_{j=0}^{[x]}(-1)^{j}\left(\begin{array}{c}
r-1 \\
j
\end{array}\right) \frac{(x-j)^{r-2}}{(r-2) !}
$$

for $r \geq 2$ and $x \geq 0$. For the proof of the above results see Takács [42]. We note that

if $r \geq 1$.

$$
\lim _{n \rightarrow \infty} \sum_{m \geq 0} \mu_{r}\left(\frac{m \sigma}{2 \sqrt{n}}\right) \frac{\sigma}{2 \sqrt{n}}=\int_{0}^{\infty} \mu_{r}(\alpha) d \alpha=2^{(r-1) / 2} \Gamma\left(\frac{r+1}{2}\right)
$$

Theorem 6: If $a=1,0<\sigma<\infty$ and $n=s d+1(s=0,1,2, \ldots)$, then

$$
\lim _{n \rightarrow \infty} P\{\xi(r) \leq x \sigma \sqrt{n} \text { for } 0 \leq r \leq \theta(i) \mid \rho(i)=n\}=F(x)
$$


where $F(x)$ is defined by (16).

Proof: The finite dimensional distributions of the process

$$
\left\{\zeta_{[n t]} /(\sigma \sqrt{n}), 0 \leq t \leq 1 \mid \rho(i)=n\right\}
$$

converge to the corresponding finite dimensional distributions of the Brownian excursion $\left\{\eta^{+}(t), 0 \leq t \leq 1\right\}$. By a theorem of Kaigh [15], [16] we have also weak convergence. A necessary and sufficient condition for weak convergence is

$$
\lim _{h \rightarrow 0} \limsup _{n \rightarrow \infty} P\left\{\max _{j-k \mid \leq n h}\left|\zeta_{j}-\zeta_{k}\right|>\epsilon \sigma \sqrt{n} \mid \rho(i)=n\right\}=0
$$

for $\epsilon>0$. Here $n=s d+1(s=0,1,2, \ldots)$ and $h>0$. See I.I. Gikhman and A.V. Skorohod [10] pp. 449-450.

By (63)

$$
\begin{gathered}
P\left\{\max _{0 \leq i \leq n} \zeta_{i}-\max _{m \geq 0} \xi(m)>\epsilon \sigma \sqrt{n} \mid \rho(i)=n\right\} \\
\leq P\left\{\max _{m \geq 0} \xi(m)>n h \mid \rho(i)=n\right\}+P\left\{\max _{|j-k| \leq n h}\left|\zeta_{j}-\zeta_{k}\right|>\epsilon \sigma \sqrt{n} \mid \rho(i)=n\right\}
\end{gathered}
$$

for any $\epsilon>0$ and $h>0$. Furthermore, if (59) holds, by (65)

$$
\begin{gathered}
P\left\{\max _{m \geq 0} \xi(m)>n h \mid \rho(i)=n\right\} \leq \sum_{m \geq 0} P\{\xi(m)>n h \mid \rho(i)=n\} \\
\leq \frac{1}{n^{r} h^{r}} \sum_{m \geq 0} E\left\{[\xi(m)]^{r} \mid \rho(i)=n\right\} \sim \frac{1}{n^{r} h^{r}}\left(\frac{\sigma \sqrt{n}}{2}\right)^{r} \sum_{m \geq 0} \mu_{r}\left(\frac{m \sigma}{2 \sqrt{n}}\right) \sim \\
\frac{1}{n^{r} h^{r}}\left(\frac{\sigma \sqrt{n}}{2}\right)^{r}\left(\sum_{\alpha \geq 0} \mu_{r}(\alpha) \Delta \alpha\right) \frac{2 \sqrt{n}}{\sigma} \sim \frac{\Gamma\left(\frac{r+1}{2}\right) \sigma^{r-1}}{2^{(r-1) / 2} h^{r} n^{(r-1) / 2}}
\end{gathered}
$$

as $n \rightarrow \infty$. Here we used the substitution $\sigma=m \sigma /(2 \sqrt{n})$. Then $\Delta \alpha=\sigma \Delta m /(2 \sqrt{n})$ and $\Delta m=1$.

If $r=2$, then by (73)

$$
\lim _{n \rightarrow \infty} P\left\{\max _{m \geq 0} \xi(m)>n h \mid \rho(i)=n\right\}=0
$$

for $h>0$. By (71) and (74)

$$
\lim _{n \rightarrow \infty} P\left\{\max _{0<i \leq n} \zeta_{i}-\max _{m \geq 0} \xi(m)>\epsilon \sigma \sqrt{n} \mid \rho(i)=n\right\}=0
$$

for any $\epsilon>0$. Consequently, by (75) and (58)

$$
\lim _{n \rightarrow \infty} P\left\{\max _{m \geq 0} \xi(m) \leq x \sigma \sqrt{n} \mid \rho(i)=n\right\}
$$




$$
=\lim _{n \rightarrow \infty} P\left\{\max _{0 \leq i \leq n} \zeta_{i} \leq x \sigma \sqrt{n} \mid \rho(i)=n\right\}=F(x) .
$$

This proves (69).

Theorem 7: If $a=1,0<\sigma<\infty$ and $n=s d+1(s=0,1,2, \ldots)$, then

$$
\lim _{n \rightarrow \infty} P\left\{\sum_{0 \leq r \leq \theta(i)}[\xi(r)]^{2} \leq x \sigma n^{3 / 2} \mid \rho(i)=n\right\}=W(x)
$$

where $W(x)$ is defined by (17).

Proof: If we use (64) and (71), then by (16) we obtain (77).

Theorem 8: If $a=1$, if $0<\sigma<\infty$, if (59) holds for $r \geq 2$ and if $n=s d+1(s=0,1,2, \ldots)$, then

$$
\lim _{n \rightarrow \infty} P\left\{\sum_{0 \leq r \leq \theta(i)} r \xi(r) \leq x \sigma n^{3 / 2} \mid \rho(i)=n\right\}=W(x)
$$

where $W(x)$ is defined by (17).

Proof: For the proof of (78) see Takács [42].

Finally, we note that it is plausible that

$$
\{2 \xi([2 \alpha \sqrt{n} / \sigma]) /(\sigma \sqrt{n}), \alpha \geq 0 \mid \rho(i)=n\} \Rightarrow\left\{\tau^{+}(\alpha), \alpha \geq 0\right\},
$$

that is, the stochastic process on the left-hand side converges weakly to the stochastic process on the right-hand side if $n \rightarrow \infty$.

\section{RANDOM ROOTED TREES}

Let us consider the queuing process introduced in Section 4. Let us suppose that the initial queue size is $i=1$, and denote by $\rho=\rho(1)$ the number of customers served in the initial busy period. If $\rho=n$, we associate a random graph with the queuing process. The graph has vertex set $(1,2, \ldots, n)$. Two vertices $r$ and $s$, where $1 \leq r<s \leq n$, are joined by an edge if and only if customer $s$ arrives during the service time of customer $r$. The random graph is evidently a tree. We designate vertex 1 as the root of the tree. If in the queuing process $\nu_{r}=i_{r}$ for $r=1,2, \ldots, n$, then necessarily

$$
i_{1}+i_{2}+\ldots+i_{n}=n-1
$$


and

$i_{1}+i_{2}+\ldots+i_{r} \geq r$ for $1 \leq r \leq n-1$.

Denote by $S_{n}$ the set of nonnegative integers $\left(i_{1}, i_{2}, \ldots, i_{n}\right)$ satisfying the conditions (80) and (81). With every sequence $\left(i_{1}, i_{2}, \ldots, i_{n}\right)$ in $S_{n}$ we associate a rooted tree. In the tree $\left(i_{1}, i_{2}, \ldots, i_{n}\right)$ two vertices $r$ and $s(1 \leq r<s \leq n)$ are joined by an edge if and only if

$$
i_{0}+i_{1}+\ldots+i_{r-1}<s \leq i_{0}+i_{1}+\ldots+i_{r}
$$

where $i_{0}=1$. In the tree $\left(i_{1}, i_{2}, \ldots, i_{n}\right)$, the root has degree $i_{1}$ and vertex $r$ $(1<r \leq n)$ has degree $i_{r}+1$.

By Theorem 1 the number of trees in $S_{n}$ is

$$
\left|S_{n}\right|=\frac{1}{n} \quad \sum_{i_{1}+i_{2}+\ldots+i_{n}=n-1} 1=\frac{1}{n}\left(\begin{array}{c}
2 n-2 \\
n-1
\end{array}\right)=C_{n-1}
$$

where $C_{0}=C_{1}=1, C_{2}=2, C_{3}=5, C_{4}=14$, are the Catalan numbers.

Let $\left\{p_{j}\right\}$ be a probability distribution on the set of nonnegative integers, that is, $p_{j} \geq 0$ for $j=0,1,2, \ldots$ and

Let

$$
\sum_{j=0}^{\infty} p_{j}=1
$$

$$
d=\operatorname{gcd}\left\{j: p_{j}>0\right\}
$$

If $S_{n}$ is not empty, that is, if $n=s d+1$ and $s$ is a sufficiently large positive integer, then let us choose a tree at random in $S_{n}$, assuming that the probability of a tree represented by $\left(i_{1}, i_{2}, \ldots, i_{n}\right)$ is

$$
p\left(i_{1}, i_{2}, \ldots, i_{n}\right)=a_{n}^{-1} p_{i_{1}} p_{i_{2}} \ldots p_{i_{n}}
$$

where

$$
a_{n}=\sum_{\left(i_{1}, i_{2}, \ldots, i_{n}\right) \in S_{n}} p_{i_{1}} p_{i_{2}} \ldots p_{i_{n}}=\frac{1}{n} \sum_{i_{1}+i_{2}+\ldots+i_{n}=n-1} p_{i_{1}} p_{i_{2}} \ldots p_{i_{n}} .
$$

If $a_{n}=0$, then (86) should be interpreted as 0 . If $P\{\rho=n\}>0$, we have

$$
P\left\{\nu_{1}=i_{1}, \nu_{2}=i_{2}, \ldots, \nu_{n}=i_{n} \mid \rho=n\right\}=p\left(i_{1}, i_{2}, \ldots, i_{n}\right) \text {. }
$$




\section{EXAMPLES FOR RANDOM ROOTED TREES}

Example 1:In the interval $(0, \infty)$, customers arrive at a counter in accordance with a Poisson process of density $\lambda$ and the service times are independent random variables each having the same exponential distribution function

In this case

$$
H(x)=\left\{\begin{array}{cc}
1-e^{-\mu x} & \text { if } x \geq 0 \\
0 & \text { if } x<0
\end{array}\right.
$$

$$
p_{j}=q p^{j}
$$

for $j=0,1,2, \ldots$ where $p=\lambda /(\lambda+\mu), q=\mu /(\lambda+\mu)$, and

$$
p\left(i_{1}, i_{2}, \ldots, i_{n}\right)=\frac{1}{C_{n-1}} \text { for }\left(i_{1}, i_{2}, \ldots, i_{n}\right) \in S_{n} .
$$

In this example, the vertices of the random tree are labeled, but we can ignore the labels, and interpret $S_{n}$ as the set of oriented (plane) rooted trees with $n$ unlabeled vertices. If we choose a tree at random in $S_{n}$, assuming that all the $\left|S_{n}\right|=C_{n-1}$ trees are equally probable, then

$$
p\left(i_{1}, i_{2}, \ldots, i_{n}\right)=\frac{1}{C_{n-1}} \text { for }\left(i_{1}, i_{2}, \ldots, i_{n}\right) \in S_{n}
$$

which is in agreement with (91).

Example 2: Let $R$ be a fixed set of nonnegative integers which always contains 0 . Let $S_{n}(R)$ be the subset of $S_{n}$ which contains all the tress $\left(i_{1}, i_{2}, \ldots, i_{n}\right)$ in $S_{n}$ for which $i_{r} \in R$ for $r=1,2, \ldots, n$, that is, if a tree belongs to $S_{n}(R)$, then the degree of the root $\in R$ and if $j$ is the degree of any other vertex of the tree, then $j-1 \in R$. Then by Theorem 3 , the number of trees in $S_{n}(R)$ is

$$
\left|S_{n}(R)\right|=\sum_{\left(i_{1}, i_{2}, \ldots, i_{n}\right) \in S_{n}(R)} 1=\frac{1}{n} \text { Coeff. of } x^{n-1} \text { in }\left(\sum_{i \in R} x^{i}\right)^{n} .
$$

Let

$$
p_{j}=p^{j} /\left(\sum_{i \in R} p^{i}\right)
$$

for $j \in R$ and $p_{j}=0$ and $j \notin R$. In this example

$$
p\left(i_{1}, i_{2}, \ldots, i_{n}\right)=\frac{1}{\left|S_{n}(R)\right|} \text { if }\left(i_{1}, i_{2}, \ldots, i_{n}\right) \in S_{n}(R) .
$$

This example can be interpreted in the following way. We consider 
$S_{n}(R)$, the set of oriented (plane) rooted trees with $n$ unlabeled vertices whenever the degrees of vertices are subject to the constraints imposed by $R$. We choose a tree at random in $S_{n}(R)$, assuming that all the possible choices are equally probable.

Example 3: Customers arrive according to a Poisson process of density $\lambda$ and the service times have unit lengths. In this case

$$
p_{j}=e^{-\lambda} \frac{\lambda^{j}}{j !} \text { for } j=0,1, \ldots
$$

and

$$
p\left(i_{1}, i_{2}, \ldots, i_{n}\right)=\frac{n !}{i_{1} ! i_{2} ! \ldots i_{n} !} \quad \frac{1}{n^{n-1}} \text { for }\left(i_{1}, i_{2}, \ldots, i_{n}\right) \in S_{n} .
$$

In this case, the procedure is equivalent to the following. We choose a tree at random in the set $S_{n}^{*}$ of rooted trees with $n$ labeled vertices, assuming that all the possible choices are equally probable. By a formula of Cayley [6], the number of such trees is $n^{n-1}$. By Theorem 1, the number of trees in $S_{n}^{*}$ is

$$
\left|S_{n}^{*}\right|=\sum_{S_{n}} \frac{n !}{i_{1} ! i_{2} ! \ldots i_{n} !}=\frac{1}{n} \sum_{i_{1}+i_{2}+\ldots+i_{n}=n-1} \frac{n !}{i_{1} ! i_{2} ! \ldots i_{n} !}=n^{n-1} .
$$

For the vertices of a tree $\left(i_{1}, i_{2}, \ldots, i_{n}\right)$ in $S_{n}$ can be labeled in

$$
\frac{n !}{i_{1} ! i_{2} ! \ldots i_{n} !}
$$

different ways. It seems (98) is the simplest proof for Cayley's formula.

Example 4: Let $R$ be again a fixed set of nonnegative integers which always contains 0 . Let $S_{n}^{*}(R)$ be the subset of $S_{n}^{*}$ which contains all the trees $\left(i_{1}, i_{2}, \ldots, i_{n}\right)$ in $S_{n}^{*}$ for which $i_{r} \in R$ for $r=1,2, \ldots, n$, that is, if a tree belongs to $S_{n}^{*}(R)$, then the degree of the root $\in R$ and if $j$ is the degree of any other vertex of the tree, then $j-1 \in R$. By Theorem 3 , the number of trees in $S_{n}^{*}(R)$ is

$$
\begin{aligned}
\left|S_{n}^{*}(R)\right|= & \sum_{\left(i_{1}, i_{2}, \ldots, i_{n}\right) \in S_{n}(R)^{i_{1} ! i_{2} ! \ldots i_{n} !}} \\
& =(n-1) ! \text { Coeff. of } x^{n-1} \text { in }\left(\sum_{i \in R} \frac{x^{i}}{i !}\right)^{n} .
\end{aligned}
$$

Let

$$
p_{j}=\left(\lambda^{j} / j !\right) /\left(\sum_{i \in R} \lambda^{i} / i !\right)
$$

for $j \in R$ and $p_{j}=0$ for $j \notin R$. In this case 


$$
p\left(i_{1}, i_{2}, \ldots, i_{n}\right)=\frac{n !}{i_{i} ! i_{2} ! \ldots . i_{n} !} \frac{1}{\left|S_{n}^{*}(R)\right|} \text { if }\left(i_{1}, i_{2}, \ldots, i_{n}\right) \in S_{n}(R) .
$$

This example can be interpreted in the following way. We consider $S_{n}^{*}(R)$, the set of rooted trees with $n$ labeled vertices whenever the degrees of the vertices are subject to the constraints imposed by the set $R$. We choose a tree at random in $S_{n}^{*}(R)$, assuming that all the possible choices are equally probable.

\section{PROBLEMS}

For a tree chosen at random in $S_{n}$ define $\tau_{n}(m)$ as the number of vertices at distance $m$ from the root. The distance of a vertex from the root is the number of edges in the path from the vertex to the root. Furthermore, define

$$
\mu_{n}=\max \left\{m: \tau_{n}(m)>0\right\}
$$

as the height of the tree,

$$
\delta_{n}=\max \left\{\tau_{n}(m): m \geq 0\right\}
$$

as the width of the tree, and

as the total height of the tree.

$$
\tau_{n}=\sum_{m \geq 0} m \tau_{n}(m)
$$

Our aim is to find the asymptotic distributions of the random variables $\tau_{n}, \mu_{n}, \delta_{n}$ and $\tau_{n}(m)$ if $m \rightarrow \infty$ and $n \rightarrow \infty$.

Let $\left\{p_{j}\right\}$ be a probability distribution on the set of nonnegative integers. Define the generating function

$$
f(z)=\sum_{j=0}^{\infty} p_{j} z^{j}
$$

for $|z| \leq 1$. We assume that $f(1)=1, f^{\prime}(1)=1, f^{\prime \prime}(1)=\sigma^{2}$ where $0<\sigma<\infty$ and $f^{(r)}(1)<\infty$ for $r>2$. Let

$$
d=\operatorname{gcd}\left\{j: p_{j}>0\right\}
$$

Let us choose a tree at random in $S_{n}$, assuming that the probability of a tree represented by $\left(i_{1}, i_{2}, \ldots, i_{n}\right)$ is

$$
p\left(i_{1}, i_{2}, \ldots, i_{n}\right)=a_{n}^{-1} p_{i_{1}} p_{i_{2}} \ldots p_{i_{n}}
$$


where $a_{n}$ is given by (87) if $S_{n}$ is not the empty set. The set $S_{n}$ is not empty if $n=s d+1$ and $s$ is a sufficiently large positive integer. In finding the asymptotic distribution of $\tau_{n}(m)$ we assume that

$$
m=[2 \alpha \sqrt{n} / \sigma]
$$

where $0<\alpha<\infty$.

Let us consider the branching process $\{\xi(r), r \geq 0\}$ defined in Section 4. The total number of individuals (total progeny) in the branching process is

and the time of extinction is

$$
\rho=\sum_{r \geq 0} \xi(r)
$$

$$
\mu=\sup \{r: \xi(r)>0\} .
$$

If extinction never happens, then $\mu=\infty$. Furthermore, let

$$
\tau=\sum_{r \geq 0} r \xi(r)
$$

that is, $\tau$ is the total number of ancestors of all the individuals in the branching process. Possibly $\tau=\infty$.

For the random trees we have

$$
\begin{gathered}
P\left\{\tau_{n}(m)=k\right\}=P\{\xi(m)=k \mid \rho=n\}, \\
P\left\{\mu_{n}=k\right\}=P\{\mu=k \mid \rho=n\}, \\
P\left\{\tau_{n}=k\right\}=P\{\tau=k \mid \rho=n\}
\end{gathered}
$$

and

$$
P\left\{\delta_{n}=k\right\}=P\left\{\max _{r \geq 0} \xi(r)=k \mid \rho=n\right\} .
$$

In proving various limit theorems for the random trees considered we assume that $a=f^{\prime}(1)=1$, that is, $\{\xi(r), r \geq 0\}$ is a critical branching process. If $a=1$, that is, $f^{\prime}(1)=1$, then $P\{\rho<\infty\}=1$. If we want to apply these limit theorems to the four examples considered in Section 8 , we should choose the parameters $p$ and $\lambda$ in such a way that the condition $a=1$ is satisfied. 


\section{THE LIMIT DISTRIBUTION OF $\tau_{n}$}

The random variable $\tau_{n}$ is the total height of a tree chosen at random in $S_{n}$. The expectation of $\tau_{n}$ has been determined by J. Riordan and N.J.A. Sloan [32] for random rooted trees with $n$ labeled vertices, and Ju. M. Voloshin [46] for random rooted trees with $n$ unlabeled vertices. See also A. Meir and J.W. Moon [27].

We can determine the distribution of $\tau_{n}$ by (115). Let us introduce the generating function

$$
\left.\Psi(z, w)=\sum_{n=1}^{\infty} P\{\rho=n\} E\left\{z^{\tau}\right\}\right\} w^{n}
$$

defined for $|z| \leq 1$ and $|w| \leq 1$. If we take into consideration that in the queuing process the number of arrivals during the first service time may be $j=0,1,2, \ldots$, we obtain that

$$
\Psi(z, w)=w f(\Psi(z, z w)) .
$$

For a given $f(z)$ we can determine the distribution and the moments of $\tau_{n}$ by (118). If $a=f^{\prime}(1)=1, \quad f^{\prime \prime}(1)=\sigma^{2}, \quad f^{(r)}(1)<\infty \quad$ for $\quad r \geq 2$, and $n=s d+1(s=0,1,2, \ldots)$, then the limit

exists for $r \geq 0$ and

$$
\lim _{n \rightarrow \infty} E\left\{\left(\frac{\sigma \tau_{n}}{2 n^{3 / 2}}\right)^{r}\right\}=M_{r}
$$

where $K_{0}=-1 / 2, K_{1}=1 / 8$ and

$$
M_{r}=K_{r} \frac{4 \sqrt{\pi} r !}{\Gamma((3 r-1) / 2) 2^{r / 2}}
$$

$$
K_{r}=\frac{3 r-4}{4} K_{r-1}+\sum_{j=1}^{r-1} K_{j} K_{r-j}
$$

for $r=2,3, \ldots$. Hence

$$
M_{r} \sim \frac{6 r}{\sqrt{2}}\left(\frac{r}{12 e}\right)^{r / 2}
$$

as $r \rightarrow \infty$ and we can conclude that there exists a distribution function $W(x)$ of a positive random variable such that

$$
\lim _{n \rightarrow \infty} P\left\{\frac{\sigma \tau_{n}}{\sqrt{4 n^{3}}} \leq x\right\}=W(x)
$$

in every continuity point of $W(x)$. The distribution function $W(x)$ is uniquely 
determined by the moments

$$
\int_{0}^{\infty} x^{r} d W(x)=M_{r}
$$

for $r=0,1,2, \ldots$. By (124) we obtain (23). For details see L. Takács [40], [42].

In the particular case where $p_{j}=(1 / 2)^{j+1}$ for $j=0,1,2, \ldots$, that is if we consider random rooted trees with $n$ unlabeled vertices, we can write that

$$
\tau_{n+1}=\frac{1}{2}\left(\sum_{i=1}^{2 n} \eta_{i}^{+}+n\right)
$$

where $\left\{\eta_{0}^{+}, \eta_{1}^{+}, \ldots, \eta_{2 n}^{+}\right\}$is a Bernoulli excursion, that is, a random walk in which $\eta_{2 n}^{+}=\eta_{0}^{+}=0$ and $\eta_{i}^{+} \geq 0$ for $0 \leq i \leq 2 n$. Since

$$
\left\{\eta_{[2 n t]}^{+} / \sqrt{n}, 0 \leq t \leq 1\right\} \Rightarrow\left\{\eta^{+}(t), 0 \leq t \leq 1\right\},
$$

if $n \rightarrow \infty$, that is, the stochastic process on the left-hand side converges weakly to the Brownian excursion, we can conclude from (123) and (124) that if $\omega^{+}$is defined by (14), we have

$$
P\left\{\omega^{+} \leq x\right\}=W(x)
$$

and

$$
E\left\{\left(\omega^{+}\right)^{r}\right\}=M_{r}
$$

for $r=0,1,2, \ldots$. See also Louchard [26].

\section{THE LIMIT DISTRIBUTION OF $\tau_{n}(m)$}

By (113) we can determine the distribution of $\tau_{n}(m)$. Let us introduce the generating function

$$
\Phi_{m}(z, w)=\sum_{n=1}^{\infty} P\{\rho=n\} E\left\{z^{\tau_{n}^{(m)}}\right\} w^{n}
$$

for $|z| \leq 1$ and $|w| \leq 1$. If we take into consideration that in the queuing process the number of customers arriving during the first service time may be $j=0,1,2, \ldots$, we obtain that

$$
\Phi_{m}(z, w)=w f\left(\Phi_{m-1}(z, w)\right)
$$

for $m=1,2, \ldots$ where 


$$
\Phi_{0}(z, w)=z \sum_{n=1}^{\infty} \frac{w^{n}}{n} P\left\{N_{n}=n-1\right\}
$$

By using (130) we can prove that if $a=f^{\prime}(1)=1, f^{\prime \prime}(1)=\sigma^{2}, f^{(r)}(1)<\infty$ for $r \geq 2$, and $n=s d+1(s=0,1,2, \ldots)$, then

$$
\lim _{n \rightarrow \infty} P\left\{\frac{2 \tau_{n}([2 \alpha \sqrt{n} / \sigma])}{\sigma \sqrt{n}} \leq x\right\}=G_{\alpha}(x)
$$

for $x>0$ where $G_{\alpha}(x)$ is the distribution function of a nonnegative random variable and is given by (26). Also

$$
\lim _{n \rightarrow \infty} E\left\{\left(\frac{2 \tau_{n}([2 \alpha \sqrt{n} / \sigma])}{\sigma \sqrt{n}}\right)^{r}\right\}=\mu_{r}(\alpha)
$$

exists for $r \geq 0$ and $\mu_{r}(\alpha)$ is defined by (28) and is given by (66) for $r \geq 2$.

The above results imply that if $\tau^{+}(\alpha)$ is the local time at level $\alpha>0$ of the Brownian excursion $\left\{\eta^{+}(t), 0 \leq t \leq 1\right\}$, then

$$
P\left\{\tau^{+}(\alpha) \leq x\right\}=G_{\alpha}(x)
$$

and

$$
E\left\{\left[\tau^{+}(\alpha)\right]^{r}\right\}=\mu_{r}(\alpha)
$$

for $r=0,1,2, \ldots$.

For random rooted trees with $n$ labeled vertices the asymptotic distribution of $\tau_{n}(m)$ was found by Stepanov [33]. See also Takács [41]. In the context of branching processes and in a different form the limit theorem (132) was found by Kennedy [19]. By his results we can conclude that

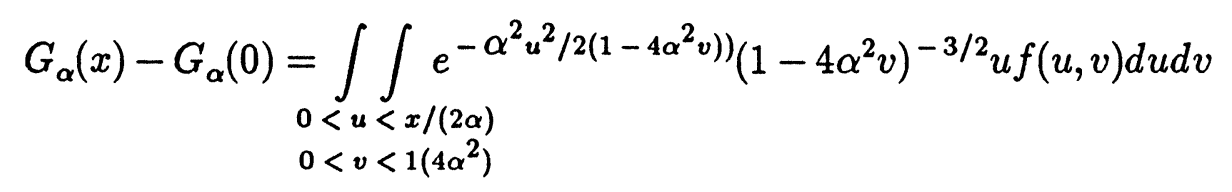

for $x>0$ and

$$
\int_{0}^{\infty} \int_{0}^{\infty} e^{-s u-w v} f(u, v) d u d v=\left[\frac{\sinh (\sqrt{2 w})}{\sqrt{2 w}}+s\left(\frac{\sinh (\sqrt{w / 2}}{\sqrt{w / 2}}\right)^{2}\right]^{-1}
$$

for $R e(s) \geq 0$ and $R e(w) \geq 0$.

If we consider the branching process $\{\xi(r), r \geq 0\}$ and use the notation

$$
\gamma(r)=\xi(0)+\xi(1)+\ldots+\xi(r)
$$


for $r \geq 0$, then by Theorem 1 we obtain that

$$
\begin{gathered}
P\{\xi(r)=k, \gamma(r)=\ell, \rho=n\}=P\{\xi(r)>0\} . \\
P\{\xi(r)=k, \gamma(r)=\ell \mid \xi(r)>0\} \frac{k}{n-\ell+k} P\left\{N_{n-\ell+k}=n-\ell\right\}
\end{gathered}
$$

if $k \geq 1$ and $n \geq \ell \geq r+k$. Thus the problem of finding the asymptotic distribution of $\tau_{n}(m)$ can be reduced to the problem of finding the asymptotic behavior of

$$
P\{\xi(r)=k, \gamma(r)=\ell \mid \xi(r)>0\}
$$

as $r \rightarrow \infty$. Kennedy [19] found that

$$
\begin{gathered}
\lim _{r \rightarrow \infty} E\left\{e^{-2(s \tau \xi(r)+w \gamma(r)) /(\sigma r)^{2}} \mid \xi(r)>0\right\} \\
=\left[\frac{\sinh (\sqrt{2 w})}{\sqrt{2 w}}+s\left(\frac{\sinh (\sqrt{w / 2}}{\sqrt{w / 2}}\right)^{2}\right]^{-1}
\end{gathered}
$$

for $\operatorname{Re}(s) \geq 0$ and $\operatorname{Re}(w) \geq 0$. In the particular case where $s=0$, this result was proved by Pakes [29] and [30]. Kennedy [19] did not provide proof of (141). He merely indicated that it can be proved by the same argument as was used by Pakes in the particular case $s=0$.

If in (139), $k=[u \alpha \sigma \sqrt{n}], \ell=\left[4 v \alpha^{2} n\right]$ and $r=[2 \alpha \sqrt{n} / \sigma]$ where $u>0$ and $0<4 \alpha^{2} v<1$, and $n \rightarrow \infty$, then by (141), we can prove (136).

\section{THE LIMIT DISTRIBUTION OF $\mu_{n}$}

Since

$$
P\left\{\mu_{n}<m\right\}=P\left\{\tau_{n}(m)=0\right\}
$$

for $n \geq 1$ and $m \geq 1$, the distribution of $\mu_{n}$ is determined by the distribution of $\tau_{n}(m)$ for $m \geq 0$.

In 1978, Kolchin [22] proved that if $a=f^{\prime}(1)=1, f^{\prime \prime}(1)=\sigma^{2}$, and $n=s d+1(s=0,1,2, \ldots)$, then

$$
\lim _{n \rightarrow \infty} P\left\{\frac{\sigma \mu_{n}}{2 \sqrt{n}} \leq x\right\}=F(x)
$$


where $F(x)$ is given by (19). Kolchin's proof is based on Theorem 1 in this paper. See also Kolchin [21].

In the particular case where $p_{j}=(1 / 2)^{j+1}$ for $j=0,1,2, \ldots$, Konovaltsev and Lipatov [23] proved (143). See also de Bruijn, Knuth and Rice [8] and Takács [34] and [38]. In the case where $p_{j}=e^{-1} / j$ ! for $j=0,1,2, \ldots$ Rényi and Szekeres [31] proved (143). See also Stepanov [33].

By (143) we expect that if (59) holds, then

$$
\lim _{n \rightarrow \infty} E\left\{\left(\frac{\sigma \mu_{n}}{2 \sqrt{n}}\right)^{r}\right\}=\mu_{r}
$$

for $r \geq 0$ where $\mu_{r}$ is given by (21). This is indeed proved in some particular cases. If $p_{j}=(1 / 2)^{j+1}$ for $j=0,1,2, \ldots$, then de Bruijn, Knuth and Rice [8] proved (144) for $r=1$ and Kemp [17] for $r \geq 1$. In some other particular cases Flajolet and Odlyzko [9] proved (144) for $r \geq 1$. For $p_{j}=e^{-1} / j$ ! $\quad(j \geq 0)$ formula (144) has not been proved yet. Rényi and Szekeres [31] state that (144) is true for $r=1$, but provide no proof.

\section{THE LIMIT DISTRIBUTION OF $\delta_{n}$}

If in the queuing process $\rho=n$, then

$$
\delta_{n}=\max \{\xi(r), r \geq 0\}
$$

and obviously,

$$
\delta_{n} \leq \max _{0 \leq i \leq n} \zeta_{i} \leq 2 \delta_{n}
$$

If $\rho=n$, then by (75)

$$
\frac{1}{\sqrt{n}}\left(\max _{0 \leq i \leq n} \zeta_{i}-\delta_{n}\right) \rightarrow 0
$$

in probability as $n \rightarrow \infty$. Thus if $\rho=n$, the random variables $\delta_{n}$ and $\max _{0 \leq i \leq n} \zeta_{i}$ have the same asymptotic distribution. Accordingly, if $a=f^{\prime}(1)=1, f^{\prime \prime}(1)=\sigma^{2}$ where $0<\sigma<\infty$ and $n=s d+1(s=0,1,2, \ldots)$, then

$$
\lim _{n \rightarrow \infty} P\left\{\frac{\delta_{n}}{\sigma \sqrt{n}} \leq x\right\}=F(x)
$$

where $F(x)$ is given by (19). Aldous [1], p. 47 has conjectured that (148) is true.

By (148) it is plausible that if (59) holds, then 


$$
\lim _{n \rightarrow \infty} E\left\{\left(\frac{\delta_{n}}{\sigma \sqrt{n}}\right)^{r}\right\}=\mu_{r}
$$

where $\mu_{r}$ is given by (21). By (60) and (146) this is true in two particular cases, namely when $p_{j}=(1 / 2)^{j+1}$ for $j=0,1,2, \ldots$ and when $p_{0}=p_{2}=1 / 2$. Odlyzko and Wilf [28] proved that if $p_{j}=e^{-1} / j$ ! for $j \geq 0$, then $E\left\{\delta_{n}\right\}=O(\sqrt{n \log n})$ as $n \rightarrow \infty$.

By (79) and (148) we can draw the conclusion that

$$
P\left\{\sup _{\alpha \geq 0} \tau^{+}(\alpha) \leq 2 x\right\}=F(x)
$$

where $\tau^{+}(\alpha)$ is defined by (15) and $F(x)$ by (19). For a direct proof of this result see Jeulin [14, p. 264].

\section{REFERENCES}

[1] Aldous, D., The continuum random tree II: An overview. In: Stochastic Analysis. Ed. by M.T. Barlow and N.H. Bingham. London Math. Soc. Lect. Notes Series. 167, Cambridge Univ. Press (1991), 23-70.

[2] Belkin, B., A limit theorem for conditional recurrent random walk attracted to a stable law. The Annals. of Math. Stat. 41 (1970), 146-163.

[3] Belkin, B., An invariance principle for conditional recurrent random walk attracted to a stable law. Zeit. für Wahrschein. und verwandte Gebiete 21 (1972), 45-64.

[4] Bertrand, J., Solution d'un problème. Compte Rendus Acad. Sci. Paris 105 (1887), 369.

[5] Bolthausen, E., On a functional central limit theorem for random walks conditioned to stay positive. The Annals of Prob. 4 (1976), 480-485.

[6] Cayley, A., A theorem on trees. Quart. J. of Pure and Appl. Math 23 (1889), 376-378. [The Collected Math. Papers of Arthur Cayley. Vol. XIII, Cambridge Univ. Press (1897), 26-28.]

[7] Chung, K.L., Excursions in Brownian motion. Arkiv för Matematik 14 (1976), 157-179.

[8] de Bruijn, N.G., Knuth, D.E., Rice, S.O., The average height of planted plane trees. Graph Theory and Computing. Ed. by R.C. Read, Academic Press, New York (1972), 15-22.

[9] Flajolet, Ph., Odlyzko, A., The average height of binary trees and other simple trees. $J$. of Compt. and Sys. Sci. 25 (1982), 171-213. 
[10] Gikhman, I.I., Skorohod, A.V., Introduction to the Theory of Random Processes. W.B. Sanders, Philadelphia 1969.

[11] Gnedenko, B.V., Studnev, Yu. P., Comparison of the effectiveness of several methods of testing homogeneity of statistical material. (Ukrianian) Dopovidi Akademii Nauk Ukrainski RSR No. 5 (1952), 359-363.

[12] Iglehart, D.L., Functional central limit theorems for random walks conditioned to stay positive. The Annals of Prob. 2 (1974), 608-619.

[13] Itô, K., McKean, Jr., H.P., Diffusion Processes and their Sample Paths. SpringerVerlag, Berlin 1965.

[14] Jeulin, Th., Application de la théorie du grossissement à l'étude des temps locaux Browniens. In: Grossissements de filtrations: exemples et applications, Ed. by Th. Jeulin and M. Yor, Lect. Notes in Math. 1118, Springer-Verlag, New York 1985, 197304.

[15] Kaigh, W.D., A conditional local limit theorem for recurrent random walk. The Annals of Prob. 3 (1975), 883-888.

[16] Kaigh, W.D., An invariance principle for random walk conditioned by a late return to zero. The Annals of Prob. 4 (1976), 115-121.

[17] Kemp, R., On the average stack size of regularly distributed binary trees. In: Automata, Lang. and Progr., Sixth Colloquium, Graz, Austria 1979. Ed. by H.A. Maurer, Lect. Notes in Comp. Sci. 71, Springer-Verlag, Berlin 1979, 340-355.

[18] Kendall, D.G., Some problems in the theory of queues. J. of the Roy. Stat. Soc. B 13 (1951), 151-185.

[19] Kennedy, D.P., The Galton-Watson process conditioned on total progeny. J. of Applied Prob. 12 (1975), 800-806.

[20] Kennedy, D.P., The distribution of the maximum Brownian excursion. J. of Appl. Prob. 13 (1976), 371-376.

[21] Kolchin, V.F., Branching processes, random trees, and a generalized scheme of arrangements of particles. Math. Notes 21 (1977), 386-394.

[22] Kolchin, V.F., Moment of degeneration of a branching process and height of a random tree. Math. Notes 24 (1978), 954-961.

[23] Konovaltsev, I.V., Lipatov, E.P., Some properties of plane rooted trees. Cybernetics 6 (1970), 660-667.

[24] Lévy, P., Sur certains processus stochastiques homogènes. Comp. Math. 7 (1940), 283339.

[25] Lévy, P., Processus Stochastiques et Mouvement Brownien. Second edition, Gauthier- 
Villars, Paris 1965.

[26] Louchard, G., The Brownian excursion area: a numerical analysis. Comp. and Math. with Appl. 10 (1984), 413-417. [Erratum: Ibid A 12 (1986), 375.]

[27] Meir, A., Moon, J.W., On the altitude of nodes in random trees. Canadian J. of Math. 30 (1978), 997-1015.

[28] Odlyzko, A.M., Wilf, H.S., Bandwidths and profiles of trees. J. of Combin. Theory B 42 (1987), 348-370.

[29] Pakes, A.G., Some limit theorems for the total progeny of a branching process. J. of Appl. Prob. 3 (1971), 176-192.

[30] Pakes, A.G., Further results on the critical Galton-Watson process with immigration. $J$. of the Aust. Math. Soc. 13 (1972), 277-290.

[31] Rényi, A., Szekeres, G., On the height of trees. J. of the Aust. Math. Soc. 7 (1967), 497-507.

[32] Riordan, J., Sloane, N.J.A., The enumeration of rooted trees by total height. $J$. of the Aust. Math. Soc. 10, (1969), 278-282.

[33] Stepanov, V.E., On the distribution of the number of vertices in strata of a random tree. Theory of Prob. and its Appl. 14 (1969), 65-78.

[34] Takács, L., Remarks on random walk problems. Publ. of the Math. Inst. of the Hung. Acad. of Sci. 2 (1957), 175-182.

[35] Takács, L., The probability law of the busy period for two types of queuing processes. Oper. Res. 9 (1961), 402-407.

[36] Takács, L., A generalization of the ballot problem and its application in the theory of queues. J. of the Amer. Stat. Assoc. 57 (1962), 327-337.

[37] Takács, L., Reflection principle. In: Ency. of Stat. Sci. 7, ed. by S. Kotz and N.L. Johnson, John Wiley and Sons, New York 1986, 670-673.

[38] Takács, L., Queues, random graphs and branching processes. J. of Appl. Math. and Simul. 1 (1988), 223-243.

[39] Takács, L., Ballots, queues and random graphs. J. of Appl. Prob. 26 (1989), 103-112.

[40] Takács, L., A Bernoulli excursion and its various applications. Adv. in Appl. Probl. 23 (1991), 557-585.

[41] Takács, L., On the distribution of the number of vertices in layers of random trees. J. of Appl. Math. and Stoch. Anal. 4 (1991), 175-186.

[42] Takács, L., Conditional limit theorems for branching processes. J. of Appl. Math. and Stoch. Anal. 4 (1991), 263-292. 
[43] Takács, L., Limit theorems for random trees. Proc. of the National Acad. of Sci. 89 (1992), 5011-5014.

[44] Takács, L., On the total heights of random rooted trees. J. of Appl. Prob. 29 (1992), 543-556.

[45] Takács, L., On the heights and widths of random rooted trees. Intern. Conf. on Random Mappings, Partitions, and Permutations, Los Angeles, Jan. 3-6, 1992. Abstract: Adv. in Appl. Prob. 24 (1992), 771.

[46] Voloshin, Ju. M., Enumeration of the terms of the object domains according to the depth of embedding. Sov. Math.-Doklady 15 (1974), 1777-1782. 


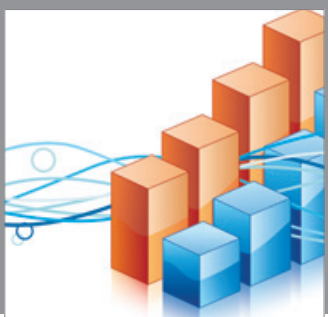

Advances in

Operations Research

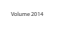

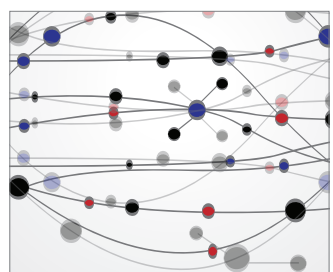

\section{The Scientific} World Journal
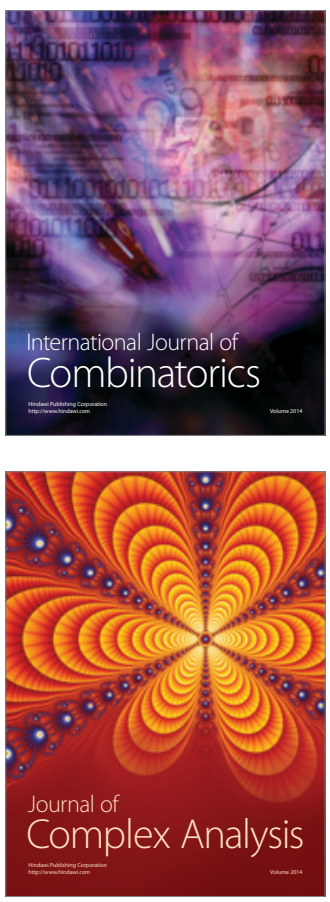

International Journal of

Mathematics and

Mathematical

Sciences
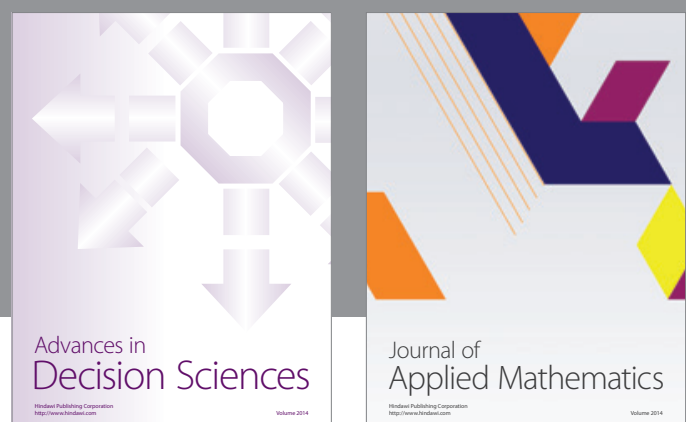

Journal of

Applied Mathematics
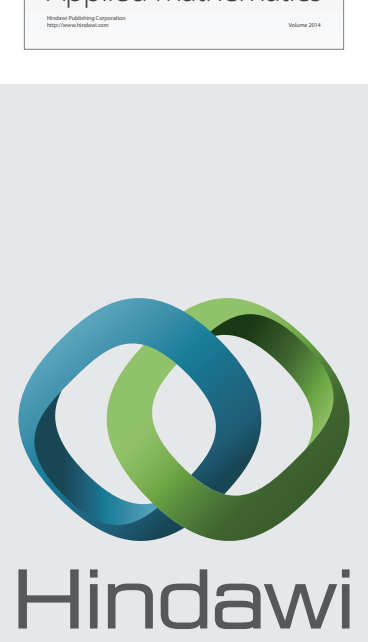

Submit your manuscripts at http://www.hindawi.com
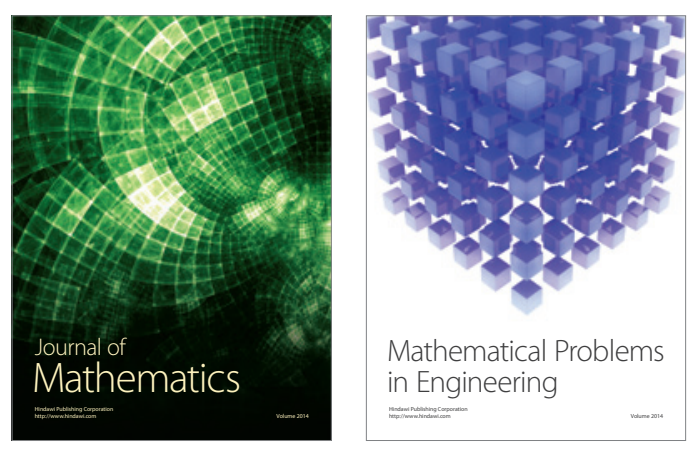

Mathematical Problems in Engineering
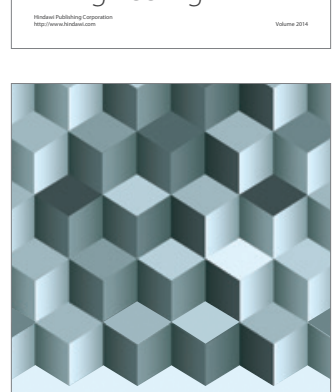

Journal of

Function Spaces
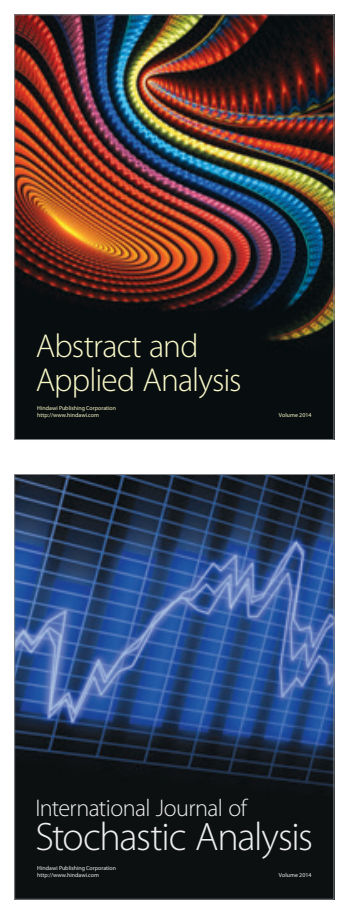

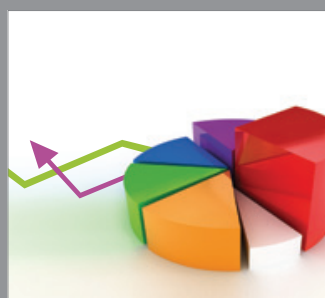

ournal of

Probability and Statistics

Promensencen
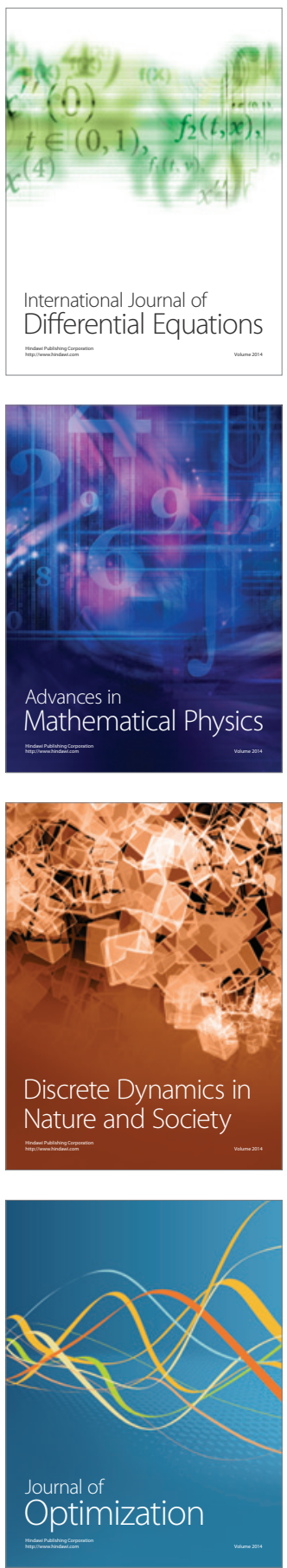